\title{
Therapeutic Effects of Ba-Duan-Jin versus Pregabalin for Fibromyalgia Treatment: Protocol for a Randomized Controlled Trial
}

\author{
Yang Yang $\cdot$ Yan-ting Li $\cdot$ Yu-ruo Sun $\cdot$ Jing Wang $\cdot$ Yang Li \\ Jin-hua Zhang · Juan Jiao · Quan Jiang (D)
}

Received: May 6, 2021 / Accepted: June 22, 2021 / Published online: July 22, 2021

(c) The Author(s) 2021

\begin{abstract}
Introduction: Fibromyalgia is characterized by multi-focal pain and is associated with fatigue, unrefreshing sleep and psychological impairment. Pregabalin is one of the most frequently used agents in fibromyalgia treatment. However, it has failed to demonstrate benefit over placebo for reducing fatigue and psychological impairment, and may cause adverse effects (e.g. somnolence, dizziness). "Ba-Duan-Jin" (BDJ) is a common form of "Qigong" exercise for health promotion in China. Growing evidence suggests that BDJ may achieve satisfactory control
\end{abstract}

Yang Yang and Yan-ting Li contributed equally to this work and share first authorship.

Y. Yang $\cdot$ J. Zhang

Psychology Department, Guang'anmen Hospital, China Academy of Chinese Medical Sciences,

Beijing, China

Y. Li · Y. Sun · Y. Li · J. Jiao $(\varangle) \cdot$ Q. Jiang $(\bowtie)$

Department of Rheumatology, Guang'anmen

Hospital, China Academy of Chinese Medical

Sciences, Beijing, China

e-mail: jiao.juan@hotmail.com

Q. Jiang

e-mail: jiang.quan@hotmail.com

J. Wang

Clinical Evaluation Centre, Guang'anmen Hospital, China Academy of Chinese Medical Sciences,

Beijing, China of fibromyalgia-related symptoms in Chinese patients. Therefore, we wish to ascertain if BDJ could overcome the disadvantages of pregabalin.

Methods: A single-blind randomized controlled trial has been designed which will recruit 104 patients with fibromyalgia (age 18-70 years) with a visual analog scale (VAS) pain score of $\geq$ $40 \mathrm{~mm}$ These patients will be randomly assigned to one of two groups: (1) BDJ group (to undertake guided BDJ exercise and take a placebo capsule) or (2) pregabalin group (to take a pregabalin capsule and receive wellness education and guided muscle-relaxation exercises). The primary endpoint will be changes in the VAS score for pain. The secondary endpoints will be changes in the score for the Revised Fibromyalgia Impact Questionnaire, Multidimensional Fatigue Inventory-20, Pittsburgh Sleep Quality Index, Beck II Depression Inventory, Perceived Stress Scale and Short Form-36 Health Survey Questionnaire. These parameters will be assessed at $0,4,8,12$ and 24 weeks of follow-up.

Planned Outcomes: Our results are expected to provide more clinical evidence for the beneficial effects of BDJ in treating fibromyalgia.

Trial Registration: NCT03797560.

Keywords: Ba-Duan-Jin; $\quad$ Fibromyalgia; Fibromyalgia-related symptoms; Pain management; Qigong 


\section{Key Summary Points}

\section{Why carry out this study?}

Many patients with fibromyalgia do not adequately respond and maintain tolerance to the first drug approved for the treatment of fibromyalgia, namely pregabalin, highlighting an unmet need for additional therapeutic agents that can effectively and safely alleviate fibromyalgia-related symptoms.

Ba-Duan-Jin" (a common form of "Qigong") can alleviate pain and other fibromyalgia-related symptoms and is associated with minimal adverse effects.

Ba-Duan-Jin may can partially "bridge" the gaps in efficacy and safety of pregabalin, but a conclusive study on its effects has not been conducted. Therefore, the purpose of this study is to address this gap in research knowledge by conducting a randomized single-blind controlled clinical trial of Ba-Duan-Jin versus pregabalin in a cohort of fibromyalgia patients with moderate pain or severe pain.

\section{What will we learn from the study?}

Our study will provide evidence as to whether Ba-Duan-Jin can bridge the gap in efficacy and safety of pregabalin and possibly become a new and efficacious treatment option for fibromyalgia.

\section{INTRODUCTION}

\section{Background}

Fibromyalgia is characterized by multi-focal pain and is associated with fatigue, unrefreshing sleep and psychological impairment [1]. These features can negatively impact quality of life and generate a significant clinical burden $[2,3]$. Fibromyalgia affects $0.2-6.6 \%$ of the world's population [4]. The percentage of patients with fibromyalgia in some cities and regions of China has been estimated to be $0.03-0.82 \%$ [5-8], but based on clinical practices in Chinese hospitals, the current incidence of fibromyalgia may be higher than that reported previously. The causes of fibromyalgia have not been clearly elucidated, but pain centralization is a known trigger [1].

Pregabalin was the first drug (and first anchor drug) approved by the US Food and Drug Administration (FDA) for fibromyalgia treatment [9], followed by approval for fibromyalgia management in China, Japan and 36 other countries [10]. The efficacy of pregabalin against the pain caused by fibromyalgia has been verified in a large body of evidence from high-quality randomized trials, systematic reviews and meta-analyses. Simultaneously, these studies have hinted towards meaningful benefits against sleep problems [10-12]. However, pregabalin is only "one piece of the puzzle" for successful management of fibromyalgia [13]. The overall therapeutic effects of pharmacotherapy are considered to be less than satisfactory because the proportion of people who achieve $\geq 50 \%$ pain relief is small, only $10-25 \%$ more than with placebo [14]. Moreover, pregabalin has not consistently demonstrated efficacy against all of the symptom domains of fibromyalgia, such as fatigue and depression. In addition, pregabalin-treated patients have a moderate-to-high incidence of adverse effects [9], and treatment discontinuation due to adverse effects occurs in $\leq 25 \%$ of patients [10].

"Qigong" is an ancient Eastern non-pharmacological therapy. It was recommended specifically for the treatment of fibromyalgia by the original European League Against Rheumatism [15]. This therapy encompasses two major factors: "Qi" and "gong". Qi refers to "vital energy" or "life force" in the entire universe and all living organisms; gong is conceptualized as the skill of regulating and balancing Qi in the body. Several forms of Qigong have been developed within different contexts. Tai Chi (the representative form of Qigong) has been an attractive option for patients with fibromyalgia. Tai Chi is a Chinese martial art practiced for defense training, health benefits and meditation. Based on the results of a study carried out 
in 2010, Wang and colleagues stated that Tai Chi is probably a safe intervention that provides significant benefits with regard to musculoskeletal pain, depression, quality of life and sleep quality; these authors reported that these benefits were sustained for 24 weeks [16]. In another study carried out in 2018, Wang and colleagues emphasized further that Tai Chi was safe and provided similar or greater improvements in fibromyalgia-related symptoms than aerobic exercise (the currently recommended basis of standard care) [17].

"Ba-Duan-Jin" (BDJ) is another form of Qigong. BDJ has been practiced widely in China for at least 800 years. Unlike Tai Chi, BDJ is characterized by simple, slow and relaxed movements to maintain physical and mental well-being rather than combat or self-defense, making it very suitable for patients with fibromyalgia. In a study on patients with fibromyalgia, we showed that, compared with conventional therapy, BDJ exercises for 12 weeks (twice per week) could result in a significant reduction in pain $(77 \%$ of participants perceived $\mathrm{a} \geq 30 \%$ reduction in pain according to a visual analog scale (VAS), fatigue, sleep disturbance and affective symptoms with few adverse effects [18]. Hence, BDJ could partially "bridge" the gaps in efficacy and safety of pregabalin, but a conclusive study has not been conducted.

\section{Objectives}

To address this gap in research knowledge, we plan to conduct a randomized single-blind controlled clinical trial of BDJ versus pregabalin in a cohort of fibromyalgia patients with moderate pain or severe pain. We also plan to create a database of empirical evidence on whether BDJ could be a treatment option among patients with fibromyalgia.

\section{METHODS}

\section{Study Design}

This study will be a 12 -week, single-center and assessor-blinded, randomized, controlled,

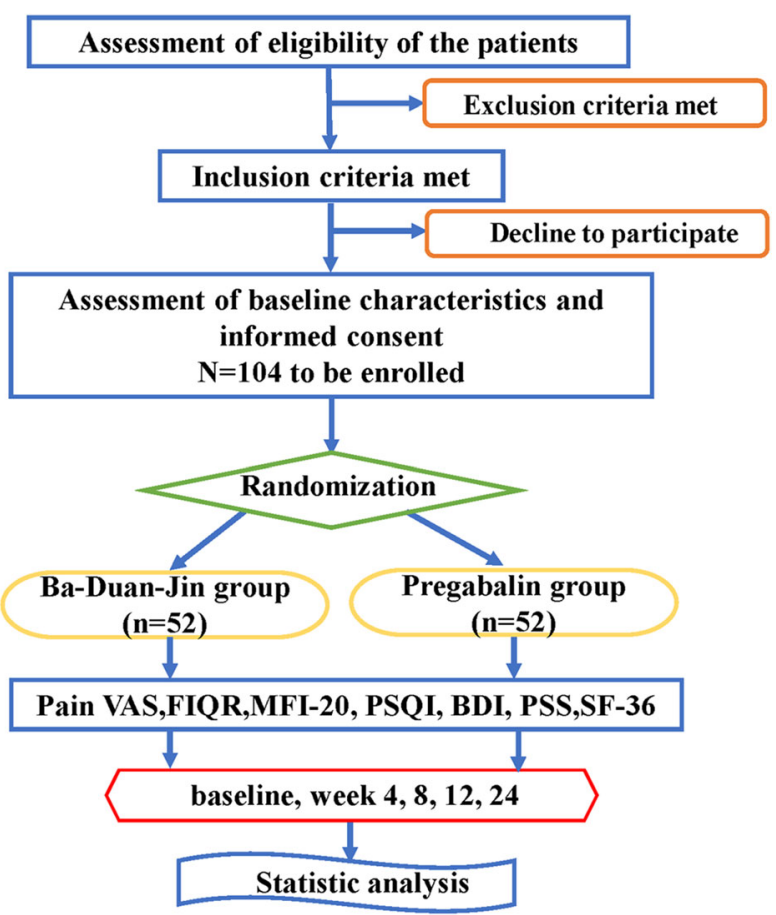

Fig. 1 Flow chart of the proposed clinical trial. $V A S$ visual analog scale, FIQR Revised Fibromyalgia Impact Questionnaire, $M F I-20$ Multidimensional Fatigue Inventory-20, PSQI Pittsburgh Sleep Quality Index, BDI Beck II Depression Inventory, PSS Perceived Stress Scale, SF-36 36-Item Short Form Health Survey Questionnaire

superiority clinical trial using two parallel groups: a BDJ group and a pregabalin group. The follow-up period will be 24 weeks. The trial will be implemented at Guang'anmen Hospital within the framework of the China Academy of Chinese Medical Sciences (Beijing, China) (Fig. 1).

\section{Participants}

The inclusion criteria and exclusion criteria are given in Table 1. Each participant will participate for 12 weeks, with pre-determined assessment time points (Table 2).

\section{Randomization}

Randomization will be controlled by an independent third party, the Clinical Evaluation Centre in Guang'anmen Hospital, after a 
Table 1 Eligibility and exclusion criteria

\begin{tabular}{ll}
\hline Inclusion criteria & Exclusion criteria \\
\hline $\begin{array}{l}\text { 1. Conform to } 1990 \text { American College of Rheumatology } \\
\text { (ACR) Research Classification Criteria for fibromyalgia }\end{array}$ & $\begin{array}{l}\text { 1. Prior experience with Ba-Duan-Jin, Tai Chi, yoga or other } \\
\text { forms of Qigong exercise in the } 12 \text { months preceding the } \\
\text { study }\end{array}$ \\
$\begin{array}{ll}\text { 2. Scores for pain intensity of }<40 \mathrm{~mm} \text { (measured by pain } \\
\text { VAS) }\end{array}$ \\
$\begin{array}{ll}\text { 3. No medication relating to fibromyalgia had been taken } 70 \text { years } & \begin{array}{l}\text { 3. Severe depression or anxiety } \\
\text { for at least 4 weeks prior to entry in the study }\end{array} \\
\text { 4. Full understanding of the research process and } & \begin{array}{l}\text { 4. Presence of any poorly controlled comorbid medical } \\
\text { conditions, such as dementia, cancer, thyroid disease, } \\
\text { nillingness to provide informed consent }\end{array} \\
\text { nulmonary disease, metabolic disease, renal disease, joint } \\
\text { disease, or other serious medical conditions limiting ability } \\
\text { to participate in the Ba-Duan-Jin }\end{array}$ \\
$\begin{array}{l}\text { 5. Pregnant or planning pregnancy within the study period } \\
\text { 6. Residing }>70 \text { miles from the research site }\end{array}$
\end{tabular}

VAS Visual Analogue Scale

baseline evaluation, using SAS v5.2.127 (SAS Institute, Cary, NC, USA).

Patients who meet the inclusion criteria must provide written informed consent. Patients will be assigned randomly to the BDJ group or pregabalin group at a ratio of $1: 1$. Information on randomized group assignments will be placed in opaque envelopes with date and signature labels printed on the seals and stored in a research office of the hospital. The baseline evaluation will be carried out 2 weeks before the start of the trial. Once participants complete the evaluations and provide written informed consent, the envelopes will be opened by a study coordinator, following which patients will be assigned to their groups. Messages containing a schedule of training sessions (including the time and address) will be sent to participants by WeChat ${ }^{\mathrm{TM}}$ v7.0.8 (Tencent, Beijing, China) or by telephone.

\section{Participant Recruitment}

Participants will be recruited mainly through outpatient clinics using posters and online publicity. This material will contain a brief introduction to the trial and the contact information of the researchers. If a patient is interested in taking part, he/she can contact one of the researchers and obtain information on the specific procedure, purpose, potential adverse effects and expected benefits of the trial.

\section{Intervention}

A brief description of the arms of the trial is shown as Fig. 2.

\section{BDJ Group (BDJ and Placebo)}

The practice of BDJ will be, in general, consistent with that described by Jiao and colleagues [18]. Upon training and guidance by an experienced instructor in $\mathrm{BDJ}$, patients will be supervised closely to practice BDJ for $50 \mathrm{~min}$ twice weekly for 12 weeks in Guang'anmen Hospital. During the first session, the participants will be provided with printed materials that will explain the theory and principles of $\mathrm{BDJ}$ and how to practice the art of BDJ. In 
Table 2 Time points and the assessed dimensions at the time points

\begin{tabular}{|c|c|c|c|c|c|c|}
\hline \multirow{2}{*}{$\frac{\text { Study period }}{\text { Timepoint (week) }}$} & \multirow{2}{*}{$\begin{array}{l}\text { Enrollment } \\
-1\end{array}$} & \multirow{2}{*}{$\begin{array}{l}\text { Allocation } \\
0\end{array}$} & \multicolumn{3}{|c|}{ Post-treatment } & \multirow{2}{*}{$\begin{array}{l}\text { Follow-up } \\
24\end{array}$} \\
\hline & & & 4 & 8 & 12 & \\
\hline \multicolumn{7}{|l|}{ Enrollment } \\
\hline Eligibility screening & $\mathrm{X}$ & & & & & \\
\hline Informed consent & $\mathrm{X}$ & & & & & \\
\hline Demographic characteristics & $\mathrm{X}$ & & & & & \\
\hline Medical history & $\mathrm{X}$ & & & & & \\
\hline Laboratory tests & $\mathrm{X}$ & & $\mathrm{X}$ & $\mathrm{X}$ & $\mathrm{x}$ & \\
\hline 2-lead electrocardiograms & $\mathrm{X}$ & & & & $\mathrm{x}$ & \\
\hline Randomization & & $\mathrm{X}$ & & & & \\
\hline Allocation & & $\mathrm{X}$ & & & & \\
\hline \multicolumn{7}{|l|}{ Interventions } \\
\hline Ba-Duan-Jin + placebo & & & & - - & - & ---- \\
\hline $\begin{array}{l}\text { Pregabalin }+ \text { wellness education } \\
+ \text { muscle relaxation exercise }\end{array}$ & & & & - - & - & --ー- - \\
\hline \multicolumn{7}{|l|}{ Assessments } \\
\hline Pain VAS & $\mathrm{X}$ & $\mathrm{X}$ & $\mathrm{X}$ & $\mathrm{X}$ & $\mathrm{X}$ & $\mathrm{X}$ \\
\hline FIQR & & $\mathrm{X}$ & $\mathrm{X}$ & $\mathrm{X}$ & $\mathrm{X}$ & $\mathrm{X}$ \\
\hline MFI-20 & & $\mathrm{X}$ & $\mathrm{X}$ & $\mathrm{X}$ & $\mathrm{X}$ & $\mathrm{X}$ \\
\hline PSQI & & $\mathrm{X}$ & $\mathrm{X}$ & $\mathrm{X}$ & $\mathrm{X}$ & $\mathrm{X}$ \\
\hline BDI-II & & $\mathrm{X}$ & $\mathrm{X}$ & $\mathrm{X}$ & $\mathrm{X}$ & $\mathrm{X}$ \\
\hline PSS & & $\mathrm{X}$ & $\mathrm{X}$ & $\mathrm{X}$ & $\mathrm{X}$ & $\mathrm{X}$ \\
\hline SF-36 & & $\mathrm{X}$ & $\mathrm{X}$ & $\mathrm{X}$ & $\mathrm{X}$ & $\mathrm{X}$ \\
\hline PGIC & & & & & $\mathrm{X}$ & $\mathrm{X}$ \\
\hline SSS & & $\mathrm{X}$ & $\mathrm{X}$ & $\mathrm{X}$ & $\mathrm{X}$ & $\mathrm{X}$ \\
\hline $50 \%$ Reduction for pain & & & $\mathrm{X}$ & $\mathrm{X}$ & $\mathrm{X}$ & $\mathrm{X}$ \\
\hline Adverse events & & & $\mathrm{X}$ & $\mathrm{X}$ & $\mathrm{X}$ & \\
\hline
\end{tabular}

$V A S$ Visual Analogue Scale, FIQR Revised Fibromyalgia Impact Questionnaire, MFI-20 Multidimensional Fatigue Inventory-20, PSQI Pittsburgh Sleep Quality Index, BDI Beck II Depression Inventory, PSS Perceived Stress Scale, SF-36 Short Form-36 Health Status Questionnaire, SSS Symptom Severity Scale

subsequent sessions, participants will practice eight forms of BDJ under the guidance and supervision of experts. Each session will involve a warm-up exercise, followed by a review of BDJ movements, breathing methods and relaxation.
Participants will also take a medium dose of placebo at bedtime each day. The initial dose will be $150 \mathrm{mg}$ per day, but will be increased to $300 \mathrm{mg}$ per day from week 2 if participants can tolerate it; if not, the dose will be reduced to 


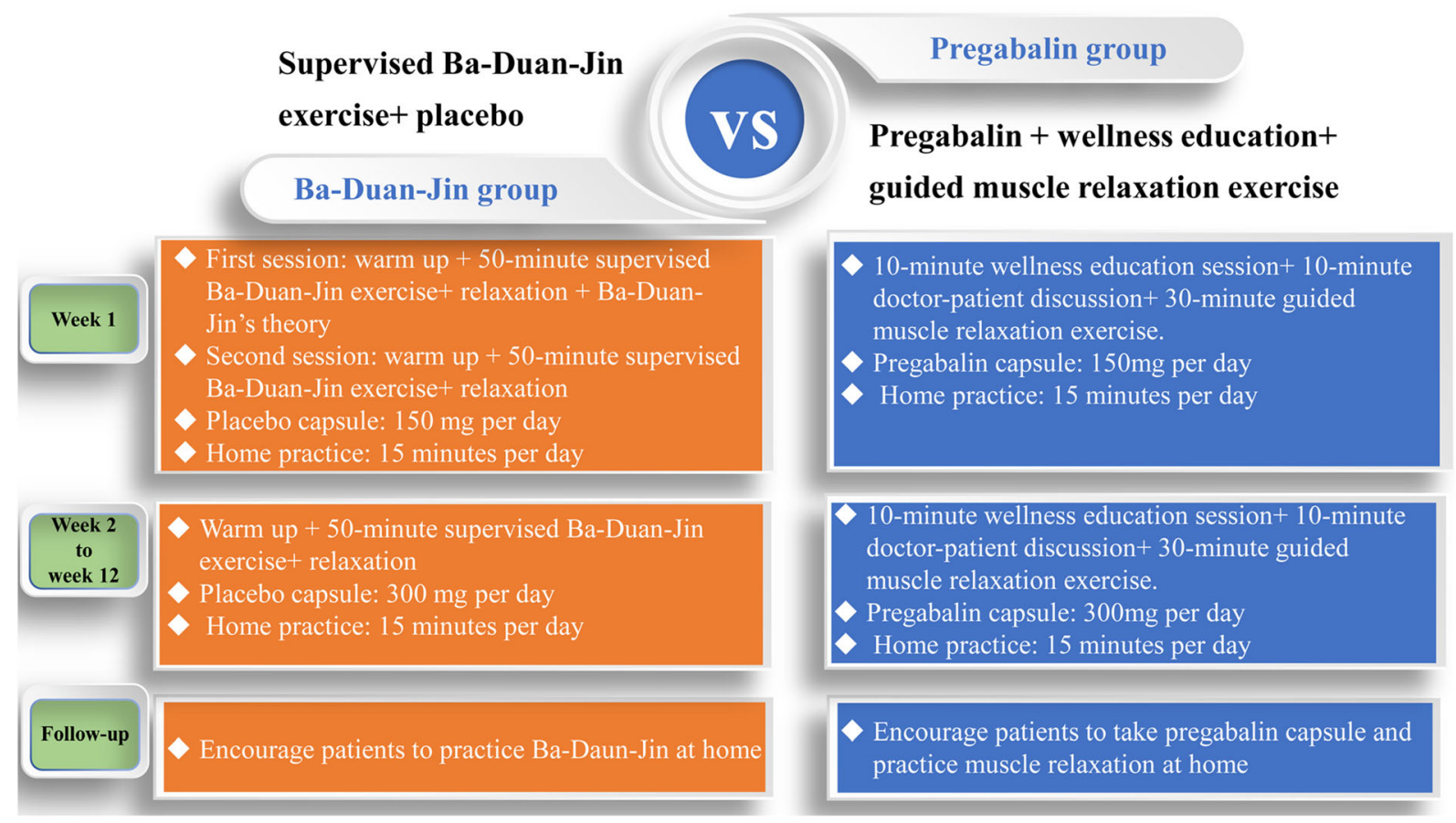

Fig. 2 Brief description of the two arms of the proposed trial

$150 \mathrm{mg}$ for the remaining 11 weeks. The placebo capsule will be prepared by the Pharmaceutical Department of Guang'anmen Hospital and will contain $150 \mathrm{mg}$ of corn starch (which is a white or slightly yellow powder).

\section{Pregabalin Group (Pregabalin, Wellness Education and Muscle Relaxation Exercises)}

A program based on wellness education and muscle relaxation exercises will be conducted, with twice-weekly sessions, each $50 \mathrm{~min}$ in duration, for 12 weeks. This program will comprise a 10-min section on wellness education, a 10-min physician-patient discussion and 30-min of guided muscle relaxation exercises. The topic of wellness education, as mentioned previously [16], will cover the diagnostic criteria for fibromyalgia, clinical symptoms, laboratory tests, treatment measures, sleep disorders, diet/ nutrition, wellness and lifestyle management. Muscle relaxation exercises will involve raising the eyebrows, closing the eyes, wrinkling the nose, opening the mouth, lifting the shoulders, making a fist, abdominal curling, lifting up the hip and stretching the thighs and calves.

The pregabalin capsule will be produced by Pfizer Pharmaceuticals (New York, NY, USA) and contain $150 \mathrm{mg}$ of pregabalin (which is a white crystalline powder). The pregabalin capsule used in the trial will be repackaged by the Pharmaceutical Department of Guang'anmen Hospital into the the same capsule as used for the placebo, with no additional components. Thus, the pregabalin and placebo capsules will be identical in terms of their appearance. The dose and timing of administration of pregabalin will be identical to those of the placebo.

Pregabalin capsules and placebo capsules will be stored separately in a locked medicine cabinet under moderate temperature and humidity conditions. The study coordinator will distribute the capsules to patients each week and record their number, distribution date and distribution quantity. Participants must sign their names on a list recording capsule distribution. Empty capsule bottles will be recycled.

The researcher will contact patients to avoid the patient missing a session, will try to overcome barriers to attendance and will encourage 
patients to attend classes to make-up for lost sessions. Participants in both groups will be encouraged to practice BDJ or muscle relaxation exercises at home for 15 min daily on the 5 days of the week that they have no guided session. Each person will be given an instructional digital video for the practice sessions at home.

When the study is completed, there will be a 24-week follow-up plan. Patients who have completed the 12-week study will be encouraged to continue BDJ or pregabalin treatment according to their grouping. A 24-week followup plan will be conducted in person or by telephone. All pharmacological and non-pharmacological treatment for fibromyalgia during the follow-up period will be recorded in detail on each report form for each patient.

\section{Blinding}

The capsule containing active pregabalin will be repackaged and retained in bottles, which will be identical to the bottles containing placebo capsules. Each bottle will be labeled with a unique code corresponding to each participant. Before group allocation, all the interventions (BDJ, pregabalin, wellness education and muscle-relaxation exercises) in this study will be discussed with each participant, with the added message that all of the treatments may benefit them. In this way, participants with fibromyalgia will be more likely to believe that the interventions in each group (which involve pharmaceutical and non-pharmaceutical therapies) will be efficacious and beneficial. They will also feel that they will be treated well irrespective of which group they are in.

Each researcher will be in charge of different assignments. One researcher will be responsible for participant recruitment and testing for tender points. Another researcher will be responsible for opening the randomized envelopes, recording the dates and signing the envelope; contacting participants; distributing medicines; evaluating the adverse effects of treatment; teaching wellness education. A third researcher will guide BDJ and muscle relaxation exercises. A fourth researcher, who is also a psychologist, will oversee the whole procedure, including completion of all questionnaires.

\section{Concomitant Care}

Participants will not be allowed to take part in other forms of exercise (e.g. Tai Chi, aerobic exercises or yoga) during the trial. Other pharmacological agents that can affect pain assessment (e.g. non-steroidal anti-inflammatory drugs or opioid analgesics) must not be taken during the trial. If patients need other treatment or concomitant care, they should contact the physician in advance, and all medications must be recorded with detailed information in the case report.

\section{Primary Outcome}

The primary outcome measure will be the change in the VAS pain score [19]. The VAS will measure fibromyalgia severity based on a 10 -point scale with " 0 " representing "no pain" and " 10 " representing "unbearable pain". The VAS score for pain will be measured at baseline as well as at weeks $4,8,12$ and 24 .

\section{Secondary Outcomes}

\section{Changes in the Revised Fibromyalgia Impact Questionnaire Score}

The Fibromyalgia Impact Questionnaire Score (FIQR) [20] is a self-designed questionnaire with ten subscales that is used to measure fibromyalgia symptoms and function domains. The FIQR includes the subscales "pain intensity", "physical function", "fatigue", "morning tiredness", "depression", "anxiety", "work dilemma" and "health well-being". Scores range from 0 to 100, with a high score indicating greater symptom burden and functional limitations compared with a low score. The FIQR will be used at five time points: baseline and at weeks 4, 8, 12 and 24, respectively. Validation of the Chinese version of the FIQR is in progress. 


\section{Changes in the Multidimensional Fatigue Inventory-20 Score}

The Multidimensional Fatigue Inventory-20 (MFI-20) was developed by Smets and colleagues in 1995 to evaluate the degree of fatigue. It covers five subscales: "mental fatigue", "general fatigue", "physical fatigue", "reduced motivation" and "reduced activity". Each subscale is composed of four items assessed with 5 -point Likert scales. Scores on each subscale range from 4 to 20 , with a high score indicating greater fatigue than a low score [21]. The MFI-20 will be implemented at five time points: baseline and weeks 4, 8, 12 and 24, respectively. Tian and Hong demonstrated that the Chinese version of MFI-20 is a reliable and valid method to measure fatigue in patients with cancer in China [22].

\section{Changes in the Pittsburgh Sleep Quality Index Score}

The Pittsburgh Sleep Quality Index (PSQI) is a well-validated and reliable evaluation tool for measuring sleep quality. It is a 19-item self-rated questionnaire for assessing sleep quality over recent months. The 19 questions are divided into seven clinical-based scores, with each subscale ranging from 0 to 3 . The total points range from 0 to 21. A high PSQI score indicates worse sleep quality than a low PSQI score [23]. The PSQI will be used at five time points: baseline and weeks 4, 8, 12 and 24, respectively. The Chinese version of the PSQI has been validated [24].

\section{Changes in the Beck II Depression Inventory Score}

The Beck II Depression Inventory (BDI-II) is used to diagnose the degree of depressive disorders. This questionnaire consists of 21 items. Each item is rated on a 4-point scale ranging from 0 to 3 , and the total score ranges from 0 to 63. A high score reflects a deeper degree of severity than a low score [25]. The BDI-II will be implemented at five time points: baseline and weeks 4, 8, 12 and 24, respectively. The Chinese version of the BDI-II has been validated [26].
Changes in the Perceived Stress Scale Score

The Perceived Stress Scale (PSS) is used to measure the perception of stress and degree of longterm stress. Scores range from 0 to 56 , with a high score indicating a higher severity of stress than a low score [27]. The PSS will be implemented at five time points: baseline and weeks $4,8,12$ and 24, respectively. The Chinese version of the PSS has been validated [28].

\section{Changes in the Score for the Patient Global Impression of Change Questionnaire}

The Patient Global Impression of Change (PGIC) questionnaire [29] is a well-established outcome measure approved by the US FDA in fibromyalgia trials. The PGIC questionnaire is used to assess overall improvement in fibromyalgia symptoms. Participants self-assess using the PGIC questionnaire, recording all changes that are perceived during the entire test. Scores range from " 1 " ("much improved") to "7" ("much worse"). The PGIC questionnaire will be used at the 12-week and 24-week followups after study completion.

\section{Changes in the 36-Item Short Form Health Survey Questionnaire Score}

The 36-Item Short Form Health Survey Questionnaire (SF-36) involves eight domains of health, and is a validated 36-item questionnaire. The scores of these subscales can be broken into two main domains: Physical Component Summary and Mental Component Summary. Scores range from 0 to 100. A high score indicates better self-perceived health status than a low score [30]. The SF-36 will be used at five time points: baseline and weeks $4,8,12$ and 24 , respectively. The Chinese version of SF36 has been verified [31].

\section{Changes in the Symptom Severity Scale Score}

The Symptom Severity Scale (SSS) is used to measure fibromyalgia symptom severity and is the sum of the severity scores of three symptoms (fatigue, waking unrefreshed and cognitive symptoms) (0-9) plus the sum (0-3) of the number of the following symptoms the patient has been bothered by that occurred during the previous 6 months: headaches $(0-1)$, pain or 
cramps in lower abdomen (0-1) and depression (0-1) [32]. The SSS will be used at five time points: baseline and weeks 4, 8, 12 and 24, respectively.

\section{The Number of Patients Achieving 50\% Reduction from Baseline in VAS for Pain} The number of patients achieving 50\% reduction from baseline in VAS for pain will be implemented at four times: weeks 4, 8, 12 and 24 , respectively.

During the study period, a standard adverse event case report will be used for monitoring adverse events at each week during capsule distribution. Each adverse event will be recorded and its severity evaluated.

Clinical laboratory evaluations (including levels of glutamic-pyruvic transaminase, glutamic oxaloacetic transaminase, creatinine and urea nitrogen) and physical examinations (tender points) will be carried out at baseline and weeks 4,8 and 12, respectively. Also, 12-lead electrocardiography will be carried out at baseline and week 12.

\section{Cohort Size}

We recently provided evidence to support the efficacy of BDJ in relieving body pain [18]. Based on a significance level of 0.05 , and a change of $30.8 \pm 22.7$ (mean \pm standard deviation) in the VAS score for pain in the BDJ-treated group [18] and a change of $10.6 \pm 13.8$ in the pregabalintherapy group [33], the threshold for a change in the VAS score for pain was set to 10 . Using PASS version 11.0 software (www.ncss.com/), we predict that 43 patients per group will be needed to detect a significant difference at $80 \%$ probability. Assuming a dropout rate of $20 \%$, we predict that 52 patients per group could achieve a significant outcome.

\section{Statistical Analyses}

Statistical analyses will be undertaken in the two treatment groups by a statistician based on intent-to-treat using SAS version 8.2 software (SAS Statistical Institute). $P<0.05$ (two-sided) will denote significance. The demographic characteristics of the BDJ group and pregabalin group will be compared by a two-sample $t$ test or Mann-Whitney test. The primary endpoint (VAS score for pain) in the two groups will be compared by the Student's $t$ test. If the data do not follow a normal distribution, a non-parametric Wilcoxon signed rank test will be used. The secondary endpoints of the two groups will be analyzed in a similar way. The change in VAS score for pain from baseline to weeks $4,8,12$ and 24 , as well as secondary outcomes for change at weeks $4,8,12$ and 24 , will be compared by linear regression or logistic regression.

\section{Compliance with Ethics Guidelines}

The study protocol has been approved by the Ethics Review Committee of the Guang'anmen Hospital, Approval Number: 2018-138-KY. All participants will be required to sign the informed consent form prior to randomization grouping. The trial has been conceived and will be carried out according to the principles that are put forward in the Declaration of Helsinki. Patients are allowed to withdraw from the study at any time without penalty.

\section{STRENGTHS AND LIMITATIONS}

\section{Strengths}

We present a plan for a robust and well-designed trial to determine the efficacy and safety of BDJ versus pregabalin for fibromyalgia management. Our study will provide new information to assist physicians and people with fibromyalgia to consider BDJ as an option in multidisciplinary management of fibromyalgia.

The acceptability of BDJ may be higher than that for pregabalin. Such acceptability is potentially influenced by the long tradition and culture of treatment with such interventions in China. To minimize the influence of pre-existing beliefs and expectations with respect to BDJ, wellness education and muscle relaxation exercises will be incorporated into the treatment regimen of the pregabalin group, and a placebo will be added to the treatment regimen of the 
BDJ group. Thus, both groups will take a capsule (placebo or pregabalin) and will attend supervised exercises twice weekly (receive BDJ exercises or muscle relaxation exercises) for 12 weeks.

Wellness education and stretching programs were used as a placebo control of Tai Chi in a study by Wang and collaborators. These authors found no significant differences in the expectations of benefit at baseline from an exercise intervention between a Tai Chi group and control group $(3.7 \pm 0.8$ and $3.9 \pm 0.7$, respectively) [16]. Muscle relaxation exercises involve the sequential tensing and relaxing of muscles. We believe that compared with stretching programs, muscle relaxation exercises, with a small stimulus and mild intensity, could be feasible for clinical trials as a control intervention.

The pregabalin dose should be based on its efficacy, specific safety concerns and patient tolerance. There is no "gold standard" for pregabalin dose in Chinese patients with fibromyalgia. One conference abstract suggested that pregabalin (300-450 mg/day) demonstrated significant improvements in pain compared with a placebo for fibromyalgia patients in China [34]. According to one study in the USA, pregabalin $(300,450$ and $600 \mathrm{mg} /$ day) resulted in greater improvements in fibromyalgia-related pain compared with that elicited by placebo. However, the dropout rate due to adverse events also increased to 16 , 22 and $26 \%$, respectively, in the dose groups and, in addition, the dropout rate among patients receiving 450 or $600 \mathrm{mg}$ /day pregabalin was higher than that in the placebo group [35], suggesting that a pregabalin dose of $300 \mathrm{mg}$ seemed to be the best compromise between efficacy and safety. Perception of efficacy may have regional disparities, so the pregabalin dose in our study will start at $150 \mathrm{mg} /$ day and then escalate to maintain a dose of $300 \mathrm{mg} /$ day 1 after 1 week later, in accordance with the experience reported in a Japanese study [36]. The time and frequency of drug use determines if a patient with chronic pain will have strong endurance [37]. Specifically, Nasser and colleagues found no comparable difference in benefit or detriment when treating fibromyalgia with $300 \mathrm{mg}$ of pregabalin twice daily or once nightly [38]. Once-nightly treatment led to a significant decrease in total patient-reported adverse events [38], and so could be a wise dosing strategy.

\section{LIMITATIONS}

The proposed study has two main limitations. First, it will be a single-center clinical trial using a small cohort of participants; second, regarding exercise therapy, it is unclear how much practice will elicit benefit.

\section{ACKNOWLEDGEMENTS}

The authors would like to thank the participants of the study.

Funding. This work was supported by the Capital Characteristic Clinical Application Research Project of Beijing Municipal Science and Technology Plan of China (Grant Number Z181100001718153). The above funding sources have no involvement in the design of the study and collection, analysis, interpretation of data and in writing the manuscript. The journal's Rapid Service Fee will be funded by Guang'anmen Hospital, China Academy of Chinese Medical Sciences.

Authorship. All named authors meet the International Committee of Medical Journal Editors (ICMJE) criteria for authorship for this article, take responsibility for the integrity of the work as a whole, and have given their approval for this version to be published.

Authors' Contributions. JJ and QJ designed the single-blind randomized controlled trial. YY, YTL, YRS, YL, JHZ and QJ conducted the research. JJ obtained funding for the research. JW was responsible for the statistical analyses. JJ, YTL and YY drafted the manuscript. All authors participated in manuscript revision and approved the final version of the manuscript. 
Disclosures. Yang Yang, Yan-ting Li, Yu-ruo Sun, Jing Wang, Yang Li, Jin-hua Zhang, Juan Jiao and Quan Jiang have nothing to disclose.

Compliance with Ethics Guidelines. The study protocol has been approved by the Ethics Review Committee of the Guang'anmen Hospital, Approval Number: 2018-138-KY. All participants will be required to sign the informed consent form prior randomization grouping. The trial has been conceived and will be carried out according to the principles that are put forward in the Declaration of Helsinki. Patients are allowed to withdraw from the study at any time without penalty.

Data Availability. Data sharing is not applicable to this article as no datasets were generated or analyzed during the current study.

Open Access. This article is licensed under a Creative Commons Attribution-NonCommercial 4.0 International License, which permits any non-commercial use, sharing, adaptation, distribution and reproduction in any medium or format, as long as you give appropriate credit to the original author(s) and the source, provide a link to the Creative Commons licence, and indicate if changes were made. The images or other third party material in this article are included in the article's Creative Commons licence, unless indicated otherwise in a credit line to the material. If material is not included in the article's Creative Commons licence and your intended use is not permitted by statutory regulation or exceeds the permitted use, you will need to obtain permission directly from the copyright holder. To view a copy of this licence, visit http:// creativecommons.org/licenses/by-nc/4.0/.

\section{REFERENCES}

1. Häuser W, Ablin J, Fitzcharles MA, et al. Fibromyalgia. Nat Rev Dis Primers. 2015;1:15022.

2. Smith HS, Harris R, Clauw D. Fibromyalgia: an afferent processing disorder leading to a complex pain generalized syndrome. Pain Physician. 2011;14(2):E217-45.

3. Chinn S, Caldwell W, Gritsenko K. Fibromyalgia pathogenesis and treatment options update. Curr Pain Headache Rep. 2016;20(4):25.

4. Marques AP, Santo ASDE, Berssaneti AA, Matsutani LA, Yuan SLK. Prevalence of fibromyalgia: literature review update. Rev Bras Reumatol Engl Ed. 2017;57(4):356-63.

5. Scudds RA, Li EKM, Scudds RJ. The prevalence of fibromyalgia syndrome in Chinese people in Hong Kong. J Musculoskelet Pain. 2016;14(2):3-11.

6. Lin L, Xiao Z, Lin SF, Chen R, Lin RR, et al. A rural population survey of soft tissue rheumatic pain in Shantou, China. Shanxi Med J. 2007;8:678-80.

7. Zang $\mathrm{CH}$, Zeng QY, Li XF, Dong HY, Zhang AL. Epidemiological study of rheumatic diseases in Taiyuan area. Chin Remedies Clin. 2007;8:597-602.

8. Zeng SY, Gong Y, Zhang YP, et al. Changes in the Prevalence of rheumatic diseases in Shantou, China, in the past three decades: a COPCORD Study. PLoS ONE. 2015;10(9): e0138492.

9. Bhusal S, Diomampo S, Magrey MN. Clinical utility, safety, and efficacy of pregabalin in the treatment of fibromyalgia. Drug Healthc Patient Saf. 2016;8: 13-23.

10. Arnold LM, Choy E, Clauw DJ, et al. An evidencebased review of pregabalin for the treatment of fibromyalgia. Curr Med Res Opin. 2018;34(8): 1397-409.

11. Arnold LM, Schikler KN, Bateman L, et al. Safety and efficacy of pregabalin in adolescents with fibromyalgia: a randomized, double-blind, placebocontrolled trial and a 6-month open-label extension study. Pediatr Rheumatol. 2016;14(1):46.

12. Derry S, Cording M, Wiffen PJ, Law S, Phillips T, Moore RA. Pregabalin for pain in fibromyalgia in adults. Cochrane Database Syst Rev. 2016. https:// doi.org/10.1002/14651858.CD011790.pub2.

13. Arnold LM, Bradley LA, Clauw DJ, Glass JM, Goldenberg DL. Multidisciplinary care and stepwise treatment for fibromyalgia. J Clin Psychiatry. 2008;69(12): e35.

14. Moore RA, Straube S, Aldington D. Pain measures and cut-offs-'no worse than mild pain' as a simple, universal outcome. Anaesthesia. 2013;68(4): 400-12.

15. Marques AP, Santo A, Berssaneti AA, Matsutani LA, Yuan SLK. Prevalence of fibromyalgia: literature 
review update. Rev Bras Reumatol (English Edition). 2017;57(4):356-63.

16. Wang $\mathrm{C}$, Schmid $\mathrm{CH}$, Rones $\mathrm{R}$, et al. A randomized trial of Tai Chi for fibromyalgia. New Engl J Med. 2010;363(8):743-54.

17. Wang C, Schmid CH, Fielding RA, et al. Effect of tai chi versus aerobic exercise for fibromyalgia: comparative effectiveness randomized controlled trial. BMJ. 2018;360:k851.

18. Jiao J, Russell IJ, Wang W, Wang J, Zhao YY, Jiang Q. Ba-Duan-Jin alleviates pain and fibromyalgiarelated symptoms in patients with fibromyalgia: results of a randomised controlled trial. Clin Exp Rheumatol. 2019;37(6):953-62.

19. Cao HXJ, Liu J. Application of visual analogue scales in assessment of symptomatic outcome data. J Trad Chin Med. 2009;50(07):600-2.

20. Bennett RM, Friend R, Jones KD, Ward R, Han BK, Ross RL. The Revised Fibromyalgia Impact Questionnaire (FIQR): validation and psychometric properties. Arthritis Res Ther. 2009;11(4):R120.

21. Lin JM, Brimmer DJ, Maloney EM, Nyarko E, Belue $\mathrm{R}$, Reeves WC. Further validation of the Multidimensional Fatigue Inventory in a US adult population sample. Popul Health Metr. 2009;7:18.

22. Tian J, Hong JS. Validation of the Chinese version of Multidimensional Fatigue Inventory-20 in Chinese patients with cancer. Support Care Cancer. 2012;20(10):2379-83.

23. Buysse DJ, Hall ML, Strollo PJ, et al. Relationships between the Pittsburgh Sleep Quality Index (PSQI), Epworth Sleepiness Scale (ESS), and clinical/ polysomnographic measures in a community sample. J Clin Sleep Med. 2008;4(6):563-71.

24. Liu XC, Tang M, Hu L, et al. Reliability and validity of the Pittsburgh sleep quality index. Chin J Psychiatry. 1996;29:103-7.

25. Arnau RC, Meagher MW, Norris MP, Bramson R. Psychometric evaluation of the Beck Depression Inventory-II with primary care medical patients. Health Psychol. 2001;20(2):2-119.

26. Yang WH, Wu D, Peng F. Application of Chinese version of Beck Depression Inventory II to Chinese first-year college students. Chin J Clin Psychol. 2012;20(6):762-4.

27. Remor E. Psychometric properties of a European Spanish version of the Perceived Stress Scale (PSS). Span J Psychol. 2006;9(1):86-93.
28. Yang TZ, Huang HT. A epidemiological study on stress among urban residents in social transition period. Chin J Epidemiol. 2003;9:11-5.

29. Dworkin RH, Turk DC, Farrar JT, et al. Core outcome measures for chronic pain clinical trials: IMMPACT recommendations. Pain. 2005;113(1-2): 9-19.

30. Elliott TE, Renier CM, Palcher JA. Chronic pain, depression, and quality of life: correlations and predictive value of the SF-36. Pain Med. 2003;4(4): 331-9.

31. Li L, Wang H, Shen Y. Development and psychometric tests of a Chinese Version of the SF-36 Health Survey Scales. Chin J Prevent Med. 2002;2: $38-42$.

32. Wolfe F, Walitt B, Perrot S, Rasker JJ, Häuser W. Fibromyalgia diagnosis and biased assessment: sex, prevalence and bias. PLoS ONE. 2018;13(9): e0203755.

33. Pauer L, Winkelmann A, Arsenault P, et al. An international, randomized, double-blind, placebocontrolled, phase III trial of pregabalin monotherapy in treatment of patients with fibromyalgia. J Rheumatol. 2011;38(12):2643-52.

34. Pauer L, Zhang F, Zhang X, Xu H, Zhang Z, Li Y. Baseline characteristics and pain response to pregabalin in a population of Chinese patients with fibromyalgia. J Pain. 2018;19(3):S78-9.

35. Arnold LM, Russell IJ, Diri EW, et al. A 14-week, randomized, double-blinded, placebo-controlled monotherapy trial of pregabalin in patients with fibromyalgia. J Pain. 2008;9(9):792-805.

36. Ohta H, Oka H, Usui C, Ohkura M, Suzuki M, Nishioka K. A randomized, double-blind, multicenter, placebo-controlled phase III trial to evaluate the efficacy and safety of pregabalin in Japanese patients with fibromyalgia. Arthritis Res Ther. 2012;14(5):R217.

37. Srivastava K, Arora A, Kataria A, Cappelleri JC, Sadosky A, Peterson AM. Impact of reducing dosing frequency on adherence to oral therapies: a literature review and meta-analysis. Patient Prefer Adherence. 2013;7:419-34.

38. Nasser K, Kivitz AJ, Maricic MJ, Silver DS, Silverman SL. Twice daily versus once nightly dosing of pregabalin for fibromyalgia: a double-blind randomized clinical trial of efficacy and safety. Arthritis Care Res (Hoboken). 2014;66(2):293-300. 